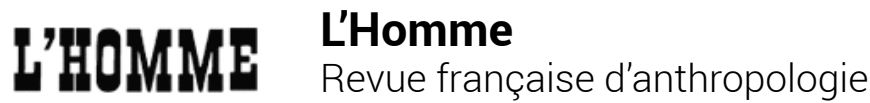

231-232| 2019

Cumulus : Hoarding, Hosting, Hospitality

\section{Cécile Leguy, ed., L'Expression de la parentalité dans les arts de la parole en Afrique}

Jean Derive

\section{OpenEdition}

Édition électronique

URL : https://journals.openedition.org/lhomme/35876

DOI : $10.4000 /$ /homme.35876

ISSN : 1953-8103

Éditeur

Éditions de l'EHESS

\section{Édition imprimée}

Date de publication : 21 novembre 2019

Pagination : 315-317

ISBN : 978-2-7132-2794-3

ISSN : 0439-4216

Référence électronique

Jean Derive, "Cécile Leguy, ed., L'Expression de la parentalité dans les arts de la parole en Afrique », L'Homme [En ligne], 231-232 | 2019, mis en ligne le 21 novembre 2019, consulté le 23 avril 2022. URL http://journals.openedition.org/lhomme/35876 ; DOI : https://doi.org/10.4000/lhomme.35876

Ce document a été généré automatiquement le 23 avril 2022.

(C) École des hautes études en sciences sociales 


\section{Cécile Leguy, ed., L'Expression de la parentalité dans les arts de la parole en Afrique}

Jean Derive

\section{RÉFÉRENCE}

Cécile Leguy, ed., L'Expression de la parentalité dans les arts de la parole en Afrique. Paris, Karthala, 2019, 248 p., notes bibliogr., ill. (« Tradition orale »).

1 L'attention portée aux relations de parenté pour comprendre le fonctionnement social a toujours été l'un des fondamentaux de l'anthropologie. La spécificité du présent volume est de se focaliser sur la "parentalité ", c'est-à-dire sur la relation parents/ enfants, étant entendu que la fonction parentale, dans sa composante nourricière aussi bien qu'éducationnelle, peut être assurée par d'autres: des membres de la famille (notamment de la branche utérine), voire, plus généralement, par différents types d'alliés, soit que ces parents par délégation se substituent aux géniteurs pour prendre seuls en charge cette fonction parentale, soit qu'ils se trouvent associés à eux pour l'assumer collectivement. Cela est particulièrement vrai pour l'Afrique, continent sur lequel porte exclusivement cet ouvrage. En effet, le régime de la polygynie, qui y est largement dominant, au moins dans la société traditionnelle, y rend plus fréquent ce type de délégation. Il est par exemple d'usage que les enfants appellent «mère » non seulement leur génitrice, mais toutes les co-épouses de celles-ci, ce qui suggère un exercice collectif de la fonction maternelle.

2 Par ailleurs, le propos de l'ouvrage, ainsi que l'explique bien, dans son introduction, Cécile Leguy, qui en a assuré la direction, est de considérer le vécu de cette relation aussi bien du point de vue des parents (biologiques ou sociaux) que de celui des enfants. En outre, les contributions ne se bornent pas à examiner la question dans les sociétés rurales dites « traditionnelles ». S'y manifeste également le souci de prendre en compte 
toutes les mutations de la société contemporaine, celles-ci - urbanisation, mixité ethnique des unions matrimoniales, migrations liées aux impératifs du travail, etc. ayant des incidences capitales sur l'exercice de la fonction parentale. Ces nouvelles données conduisent parfois à des types de délégation inédits, qui n'étaient pas prévus dans l'étiquette de la tradition et qui peuvent être sources de tension entre tradition et modernité.

3 Mais l'originalité principale de ce recueil d'études est que cette question de la parentalité est abordée non pas seulement à partir d'observations de terrain sur le fonctionnement concret de la relation parentale, mais aussi et d'abord sur sa représentation et sa mise en scène dans les arts de la parole, que ceux-ci relèvent d'un répertoire patrimonial oralement consigné (contes, épopées, chants cérémoniels) ou d'une création plus moderne (romans, chansons urbaines inspirées de rythmes contemporains).

4 Les sociologues et les critiques littéraires savent depuis toujours que les évocations des comportements sociaux dans la littérature orale ou écrite ne sont pas le reflet de la réalité sociale. Il peut y avoir un hiatus important entre ce qui est représenté et ce qui a lieu dans les faits. La littérature, entendue au sens large, a coutume, en effet, soit d'idéaliser ces comportements, en cherchant à établir, à des fins d'éducation, le canon d'une norme destinée à préparer des citoyens conformes à l'idéal social, soit d'exprimer par rapport à ces canons des positions plus subversives et revendicatives qui sont un moteur d'évolution de la société.

5 Par exemple, le rôle des parents, géniteurs ou sociaux, dans l'arrangement d'un mariage imposé à leurs enfants ou dans l'excision de leur fille, se trouve de plus en plus contesté dans le répertoire des contes et des chants traitant de ces thèmes. De même, le statut de "mère " attribué en principe à toutes les co-épouses de la génitrice est souvent dépeint dans la littérature orale sous un jour négatif, du fait de la jalousie qui règne souvent entre elles. Cette mère sociale seconde y prend donc fréquemment les traits d'une marâtre-bourreau, martyrisant l'enfant qu'elle n'a pas conçu, cela sans doute moins afin de dénoncer une pratique réelle que pour la prévenir et conjurer cette tentation. Plusieurs études du présent livre s'attachent dès lors à examiner les relations parentales entre représentations et réalité des usages, en mesurant la distance éventuelle entre les deux. Sont aussi prises en compte les répercussions que peuvent avoir ces mises en scène textuelles dans les arts de la parole sur les comportements existants, qu'elles contribuent parfois à faire évoluer par leurs revendications subversives.

6 L'ouvrage comprend neuf études distinctes. Trois d'entre elles portent sur l'Afrique centrale (Cameroun, Centrafrique, Gabon), tandis que les six autres se rapportent à l'Afrique de l'Ouest.

7 Pour l'Afrique centrale, Marie-Rose Abomo-Maurin s'intéresse à un roman d'Ahanda Essomba, Le Fruit défendu (1975), dont l'action se déroule en milieu bulu-beti-fang, qui s'étend sur plusieurs pays jusqu'au sud du Cameroun. Ce roman met en lumière le hiatus existant entre le fonctionnement traditionnel des règles de parenté dans cette société et l'idée que peut en avoir un jeune citadin, issu de ce groupe mais élevé en ville en dehors de ce système réglementaire dont il ignore tout. En séduisant malgré elle une lointaine cousine venue faire ses études en ville et confiée à son père, ce garçon, incapable de mesurer l'étendue du réseau de parenté bulu-beti-fang, se rend sans le comprendre coupable d'inceste aux yeux de sa société d'origine. Paulette Roulon-Doko, 
quant à elle, examine le rôle fondamental de l'oncle utérin comme père de substitution privilégié dans un échantillon de contes gbaya de la République centrafricaine. AngèleChristine Ondo, enfin, pointe l'image démesurée conférée à la parentalité dans un genre épique, le mvet ekang, au Gabon.

Pour l'Afrique occidentale, cinq contributions reviennent sur les composantes de l'expression de la parentalité dans la littérature orale patrimoniale selon différents genres :

- les chants d'excision chez les Tagba de Mahon au Burkina Faso (Edwidge Traore);

- le conte merveilleux: Klaus Hamberger étudie trente-deux versions d'un conte-type largement répandu en Afrique de l'Ouest, "L'enfant jeté», pour dresser une cartographie complexe des relations parentales impliquées dans les diverses déclinaisons de la matrice de ce récit, suivant les systèmes parentaux en cours dans les sociétés où il a été recueilli;

- les poèmes d'éloge des enfants par leur mère chez les Zarma du Niger (Sandra Bornand) : cette contribution met en exergue le fait que les règles de bienséance en usage dans cette société contraignent les mères à trouver des stratégies pour exprimer ces éloges de manière indirecte ;

- les rituels de nomination de l'enfant chez les Bwa du Mali (Cécile Leguy), analysés en tant qu'énoncés performatifs pour l'avenir de la relation parentale ;

- les poèmes wolof $\mathrm{du}$ Sénégal (Abdoulaye Keïta) traitant du caractère fondamentalement sacré de cette relation parentale ;

- la littérature néo-orale à travers les images maternelles représentées dans la chanson populaire burkinabé contemporaine (Alice Degorce).

9 L'ouvrage réunit donc plusieurs centres d'intérêt. Il est en premier lieu une source riche d'informations sur le fonctionnement de la parentalité et la représentation qui en est donnée dans les genres littéraires oraux ou écrits de plusieurs sociétés africaines, caractéristiques de la situation idéologique dominante à cet égard sur l'ensemble du continent. Indépendamment de ce thème particulier, il offre une nouvelle occasion d'éclairer les rapports entre l'image de la société telle qu'elle est montrée dans la littérature, qu'elle soit orale, néo-orale ou écrite, et la réalité des pratiques sociales. C'est pourquoi il est susceptible d'intéresser aussi bien les anthropologues que les critiques littéraires. 